\title{
Bridging the Gap between Polymer Melts and Solutions in Extensional Rheology
}

\author{
Huang, Qian; Hengeller, Ludovica; Alvarez, Nicolas J.; Hassager, Ole
}

Published in:

Macromolecules

Link to article, DOI:

10.1021/acs.macromol.5b00849

Publication date:

2015

Document Version

Peer reviewed version

Link back to DTU Orbit

Citation (APA):

Huang, Q., Hengeller, L., Alvarez, N. J., \& Hassager, O. (2015). Bridging the Gap between Polymer Melts and Solutions in Extensional Rheology. Macromolecules, 48(12), 4158-4163.

https://doi.org/10.1021/acs.macromol.5b00849

\section{General rights}

Copyright and moral rights for the publications made accessible in the public portal are retained by the authors and/or other copyright owners and it is a condition of accessing publications that users recognise and abide by the legal requirements associated with these rights.

- Users may download and print one copy of any publication from the public portal for the purpose of private study or research.

- You may not further distribute the material or use it for any profit-making activity or commercial gain

- You may freely distribute the URL identifying the publication in the public portal

If you believe that this document breaches copyright please contact us providing details, and we will remove access to the work immediately and investigate your claim 


\title{
Bridging the gap between polymer melts and solutions in extensional rheology
}

\author{
Qian Huang ${ }^{1}$, Ludovica Hengeller ${ }^{1}$, Nicolas J. Alvarez ${ }^{2}$, and Ole Hassager ${ }^{1}$ \\ ${ }^{1}$ Department of Chemical and Biochemical Engineering, Technical University of Denmark, DK-2800 Kgs. \\ Lyngby, Denmark \\ ${ }^{2}$ Department of Chemical and Biological Engineering, Drexel University, PA-19104, Philadelphia, USA
}

(Dated: May. 22, 2015)

\begin{abstract}
Since its inception, the tube model of polymer dynamics has undergone several modifications to account for observed experimental trends. One trend that has yet to be captured by a modified version of the tube model is the observed experimental difference between concentrated polymer solutions and polymer melts. We compare the nonlinear extensional rheology of a series of polystyrene solutions with wide concentration range between $10 \%$ and $100 \%$ (melt) in order to determine the key missing physics that can account for dilution effects. All the solutions studied have the same number of entanglements per chain, and are diluted in the same solvent (oligomeric styrene). We show that the difference in nonlinear rheological behavior between polystyrene melts reported by Bach et al.[1], and polystyrene solutions reported by Bhattacharjee et al. [2] and Sridhar et al. [3], can be bridged by changing the polystyrene concentration. The results presented represent a unique benchmark for all future modifications to the tube model.
\end{abstract}

\section{Introduction}

The tube model [4] is the most prolific model used to date to describe the dynamics of entangled polymer systems. Since its inception, several modifications have been made to the original theory in order to account for the observed experimental trends. For example, modifications including the concept of constraint release (CR)[5] and contour length fluctuations (CLF) [6], were introduced to the tube model in order to quantitatively address experimentally observed aspects in linear viscoelastic (LVE) properties for linear entangled polymer melts and solutions. More mechanisms such as chain stretch [7] and convective constraint release (CCR) [8] were introduced to account for non-linear behavior of dilute entangled polymer solutions in extensional flow, such as the polystyrene solutions with concentrations lower than $20 \%$ reported by Bhattacharjee et al. [2] and Sridhar et al. [3].

Despite the introduction of the above mentioned mechanisms and physics, the tube model does not correctly predict the non-linear extensional rheology of polymer melts [1]. Bach and coworkers were the first to show that polystyrene melts in extensional rheology show significant extensional strain rate thinning [1], which was the exact opposite trend reported for polystyrene solutions by Bhattacharjee et al. [2] and Sridhar et al. [3]. Polystyrene solutions show significant extensional strain rate thickening at the same Weissenberg numbers. At present there are no modifications to the tube model that can account for this difference. This has left many open questions and a search for new physics that will correctly predict both the observed experimental results for polymer solutions as well as polymer melts.

The focus of our recent work has been to attempt to determine experimentally why polymer solutions and melts behave differently, i.e. experimentally determine the missing physics. The concept of universality in polymer dynamics underlies the fundamental tube model theory and states that any two systems with the same physical parameters must exhibit identical linear and nonlinear dynamics. Using 
this fundamental understanding, Huang and coworkers $[9,10]$ compared polystyrene solutions (higher concentration than previously studied) and melts with the same number of entanglements. Congruent with universality, the data shows that the linear rheological behavior of both polystyrene solution and melt are identical when the moduli are scaled by the plateau modulus and frequency is normalized by the inverse relaxation time of one entanglement. Contrary to universality, the polymer melt and solution behave very differently in nonlinear extensional rheology even though the number of entanglements are identical. To complicate the issue further, the higher concentration solutions using a different molecular weight polystyrene in the Huang et al. study show very different nonlinear behavior than Bhattacharjee et al. [2] and Sridhar et al. [3]; suggesting that the nonlinear behavior of concentrated solutions depend on concentration and/or molecular weight.

It would appear that these recent findings have complicated the issue rather than narrow down the missing physics. However, there is an important parameter, which accounts for the flexibility of the polymer chain, which fundamentally changes with dilution/concentration. The flexibility of a polymer chain, also known as the finite extensibility of the chain, $\lambda_{\max }$, increases upon dilution; meaning that a polymer chain can in principle be stretched more in a dilute environment than a concentrated one. Therefore, from melt [1], to concentrated solution [9, 10], and to dilute solutions [2, 3] there is a steady increase in the flexibility of the chain. The three data sets in fact show that the amount of extension rate thickening increases with decreasing polymer concentration, which is in-line with increasing flexibility. This suggests that the degree of extension rate thickening in a polymer sample (solution or melt) increases with increasing flexibility and is one half of the key physics that must be properly accounted for in the tube model to accurately predict polymer dynamics. The other half of the key physics must account for the stretch/orientation induced reduction of friction as proposed by Yaoita et al. [11] and Ianniruberto et al. [12].

The goal of the present work is to determine the dependency of nonlinear extensional dynamics on the flexibility of the chain and bridge the gap between polymer melts and dilute polymer solutions. We show that the steady-state extensional viscosity of polystyrene solutions changes from stretch rate thinning to stretch rate thickening by decreasing the polystyrene concentration, i.e. increasing chain flexibility. When the concentration is low enough and the flexibility of the chain large enough, the data can be described by the modified tube model that was used to compare to the dilute solutions of the Sridhar group. In addition to experimentally explaining the difference between polymer melts and solutions, the data shown here provides a unique bench mark for all future modifications to the tube model where all parameters are fixed except chain flexibility.

\section{Experimental Details}

Polystyrenes of four different molecular weight, PS-545k, PS-900k, PS-1760k and PS-3280k, were separately diluted in an oligomeric styrene, OS-4k, to make four polystyrene solutions. PS-545k was synthesized by living anionic polymerization and is identical to the material used in Ref. [9]. PS-900k was bought from ChemcoPlus. PS-1760k, PS-3280k and OS-4k were bought from Polymer Standards Service (PSS). All the purchased materials were checked by size exclusion chromatography (SEC) to confirm the molecular weight. Table 1 summarizes the weight average molecular weight $M_{w}$ and the polydispersity index $P D I$ provided by the suppliers as well as obtained from our SEC.

Table 1: The molecular weight of the polystyrenes and the styrene oligomer

\begin{tabular}{lcccc}
\hline \hline Sample Name & $\begin{array}{c}M_{w}[\mathrm{~g} / \mathrm{mol}] \\
\text { (from suppliers) }\end{array}$ & $\begin{array}{c}P D I \\
\text { (from suppliers) }\end{array}$ & $M_{w}[\mathrm{~g} / \mathrm{mol}]$ & $P D I$ \\
\hline PS-545k & - & - & 545000 & 1.12 \\
PS-900k & 900000 & 1.10 & 910000 & 1.16 \\
PS-1760k & 1760000 & 1.04 & 1880000 & 1.13 \\
PS-3280k & 3280000 & 1.11 & 3140000 & 1.38 \\
OS-4k & 4330 & 1.04 & 3630 & 1.06 \\
\hline \hline
\end{tabular}


Table 2: The components and the glass transition temperatures of the polystyrene solutions. Data for PS-285k is taken from Ref. [9] and data for PS-545k/4k-52 is taken from Ref. [10].

\begin{tabular}{lccc}
\hline \hline Sample Name & Components & $\phi[$ wt $\%$ of PS $]$ & $T_{g}\left[{ }^{\circ} \mathrm{C}\right]$ \\
\hline PS-285k & $285 \mathrm{k}$ & $100 \%$ & 107.5 \\
PS-545k/4k-52 & $545 \mathrm{k}+4 \mathrm{k}$ & $52 \%( \pm 1 \%)$ & 98.6 \\
PS-900k/4k-33 & $900 \mathrm{k}+4 \mathrm{k}$ & $33 \%( \pm 1 \%)$ & 92.1 \\
PS-1760k/4k-18 & $1760 \mathrm{k}+4 \mathrm{k}$ & $18 \%( \pm 1 \%)$ & 89.0 \\
PS-3280k/4k-13 & $3280 \mathrm{k}+4 \mathrm{k}$ & $13 \%( \pm 1 \%)$ & 88.4 \\
\hline \hline
\end{tabular}

The PS solutions were prepared using the same procedure described in Ref. [9]. The concentrations of all the solutions were confirmed by the peak areas of the bimodal curve in SEC. Table 2 summarizes the components, the weight fraction $\phi$ and the glass transition temperature $T_{g}$ of the solutions. The density of the polystyrenes and the styrene oligomer is assumed independent of molecular weight, and therefore the the weight fraction is equivalent to the volume fraction.

The concentrations in Table 2 were chosen in order to keep the number of entanglements per chain $Z$ the same for all the samples (higher molecular weight requires more dilution). We showed in the previous study [9] that samples with identical $Z$ have the same linear viscoelastic properties. Figure 1 shows the linear rheology at the reference temperature $130{ }^{\circ} \mathrm{C}$ for all the samples including a polystyrene melt (PS-285k) with the same $Z$. For the solutions with the concentration lower than $50 \%$, influence of the solvent viscosity is considered and the values of the loss modulus $G^{\prime \prime}$ in the figure are corrected by

$$
G_{\text {corr }}^{\prime \prime}=G^{\prime \prime}-\phi_{s} \eta_{s} \omega
$$

where $\phi_{s}$ is the volume fraction of OS- $4 \mathrm{k}$ and $\eta_{s}=248 \mathrm{~Pa} . \mathrm{s}$ is the viscosity of OS- $4 \mathrm{k}$ at $130{ }^{\circ} \mathrm{C}$. LVE data for OS-4k can be found in the appendix. The lines in the figure represent the Baumgaertel-SchausbergerWinter (BSW) relaxation spectrum [13], which will be used to predict the linear behavior in uniaxial constant strain rate extensional flow. The values of the BSW parameters, $n_{e}, n_{g}, G_{N}^{0}, \tau_{c}$ and $\tau_{m}$, are listed in Table 3. For nearly monodisperse melts and solutions, the values of $n_{e}$ and $n_{g}$ are fixed to 0.23 and 0.70 , respectively [14]. The adjustable parameters, i.e. the plateau modulus $G_{N}^{0}$, the relaxation time of one entangled segment $\tau_{c}$ and the maximum relaxation time of the polymer chain $\tau_{m}$, are found by least squares fitting to the LVE data. Details about the meaning of the BSW parameters and their relation to tube model parameters are explained in Ref. [9]. It is found that the values of $G_{N}^{0}$ reported in Table 3 follow the scaling rule of $G_{N}^{0} \propto \phi^{1+\alpha}$ with $\alpha=1$. Details can be found in the appendix. The number of entanglements per chain $Z$ for the solutions is obtained from the relation $\tau_{m} / \tau_{c} \propto Z^{3.4}$ and $Z=41.0$ for PS-545k [9]. Values of $Z$ are also listed in Table 3. Figure 2 compares the LVE data under the dimensionless parameters where the angular frequency $\omega$ is normalized by $1 / \tau_{c}$ and the storage modulus $G^{\prime}$ and loss modulus $G^{\prime \prime}$ are normalized by $G_{N}^{0}$. A master curve is obtained as shown in Figure 2, confirming all the samples have the same number of entanglements per chain.

Furthermore, the LVE data in Figure 1 was measured at different temperatures and shifted to the reference temperature using the time-temperature superposition procedure. Although the polystyrene solutions have different $T_{g}$, it is found that the temperature shift factor $a_{T}$ for each solution can be described by one Williams-Landel-Ferry (WLF) equation, which agrees with the observation reported in Ref.[15]. Details can be found in the appendix.

The uniaxial extensional stress was measured by a filament stretching rheometer (FSR) [16]. Prior to making a measurement, the samples were molded into cylindrical test specimens with a fixed radius of either $R_{0}=2.7 \mathrm{~mm}$ or $R_{0}=4.0 \mathrm{~mm}$. The initial length $L_{0}$ of the cylindrical test specimens was controlled by the addition of a given mass of the sample into the mold. The aspect ratio $\Lambda_{0}=L_{0} / R_{0}$ is between 0.48 and 0.59 . The samples were pressed at $140-150{ }^{\circ} \mathrm{C}$ and annealed at the same temperature under vacuum until the polymer chains were completely relaxed.

All the samples were pre-stretched to a radius $R_{p}$ ranging from $1.2 \mathrm{~mm}$ to $3 \mathrm{~mm}$ at elevated temperatures, $150-160^{\circ} \mathrm{C}$ prior to the extensional experiments. After pre-stretching, the temperature was decreased to $130{ }^{\circ} \mathrm{C}$ for the extensional stress measurements. The samples were kept under a nitrogen atmosphere at all times. After the experiments, the samples were checked by SEC to ensure that no 


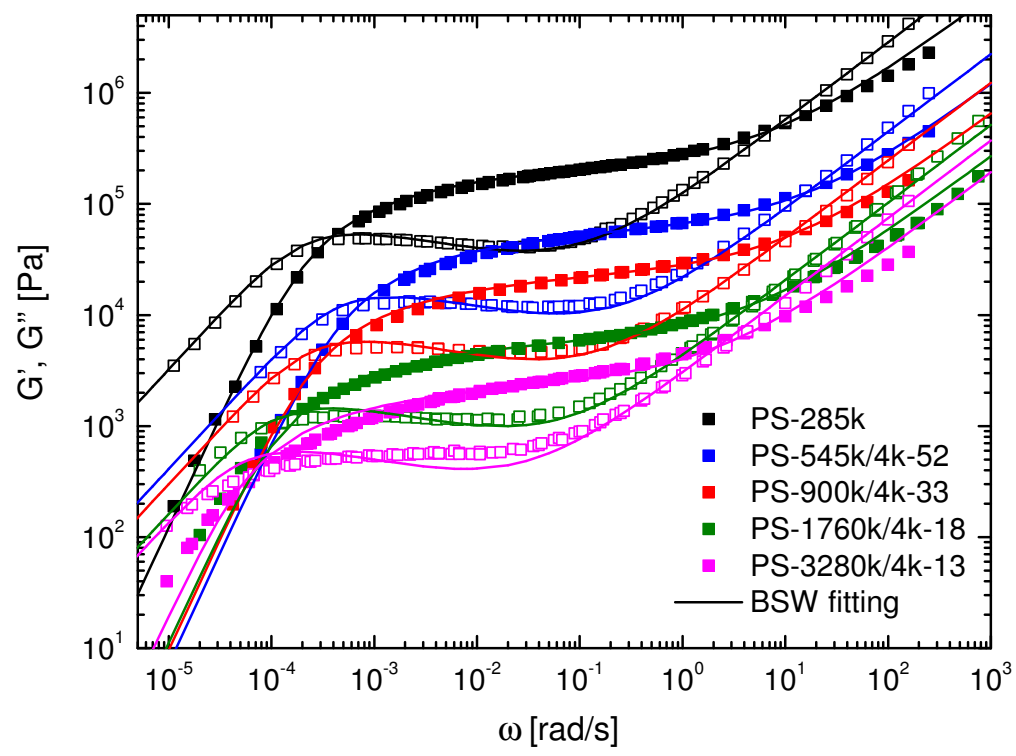

Figure 1: Storage modulus $G^{\prime}$ and loss modulus $G^{\prime \prime}$ as a function of angular frequency $\omega$ fitted with the BSW spectrum at $130{ }^{\circ} \mathrm{C}$ for the polystyrene solutions. Data for PS-285k is taken from Ref. [9]; Data for PS-545k/4k-52 is taken from Ref. [10].

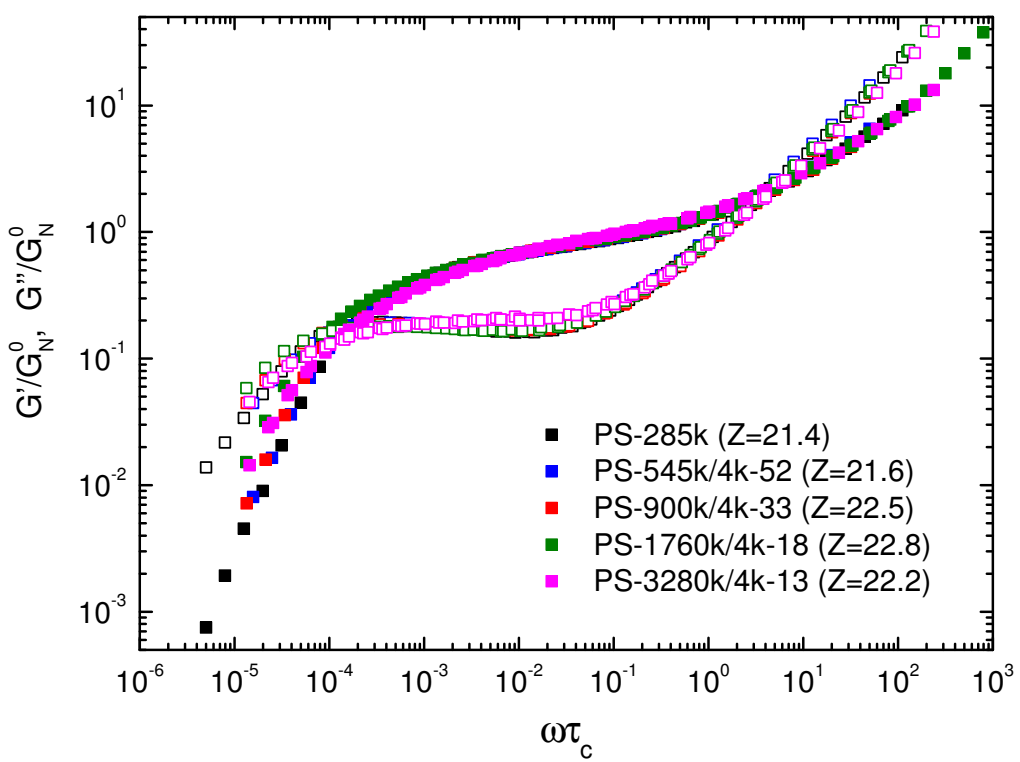

Figure 2: Normalized LVE data for polystyrene solutions from Figure 1. 
Table 3: Material properties obtained from the BSW spectrum at $130^{\circ} \mathrm{C}$. Data for PS-285k is taken from Ref. [9]; Data for PS-545k/4k-52 is taken from Ref. [10].

\begin{tabular}{lcccccccc}
\hline \hline Sample Name & $n_{e}$ & $n_{g}$ & $G_{N}^{0}[\mathrm{~Pa}]$ & $\tau_{c}[\mathrm{~s}]$ & $\tau_{m}[\mathrm{~s}]$ & $Z$ & $\lambda_{\max }$ & $N_{e}$ \\
\hline PS-285k & 0.23 & 0.7 & 252040 & 0.444 & 6846 & 21.4 & 4.7 & 22.1 \\
PS-545k/4k-52 & 0.23 & 0.7 & 68900 & 0.200 & 3180 & 21.6 & 6.5 & 42.3 \\
PS-900k/4k-33 & 0.23 & 0.7 & 27320 & 0.320 & 5844 & 22.5 & 8.2 & 67.2 \\
PS-1760k/4k-18 & 0.23 & 0.7 & 6850 & 0.661 & 12615 & 22.8 & 11.1 & 123.2 \\
PS-3280k/4k-13 & 0.23 & 0.7 & 2790 & 1.511 & 26373 & 22.2 & 13.0 & 169.0 \\
\hline \hline
\end{tabular}

degradation or concentration changes occurred during testing. No changes were observed for all the samples except PS-3280k with showing a shift from PDI 1.38 to 1.77, signifying degradation of the long chains during experiments.

The samples were subject to constant uniaxial Hencky strain rate $\dot{\epsilon}$, and the stress at the mid-filament plane was measured as a function of time. This is denoted as extensional stress growth coefficient and is defined as $\bar{\eta}^{+}=\left\langle\sigma_{z z}-\sigma_{r r}\right\rangle / \dot{\epsilon}$. Details are given in Ref. [9].

\section{Results and discussions}

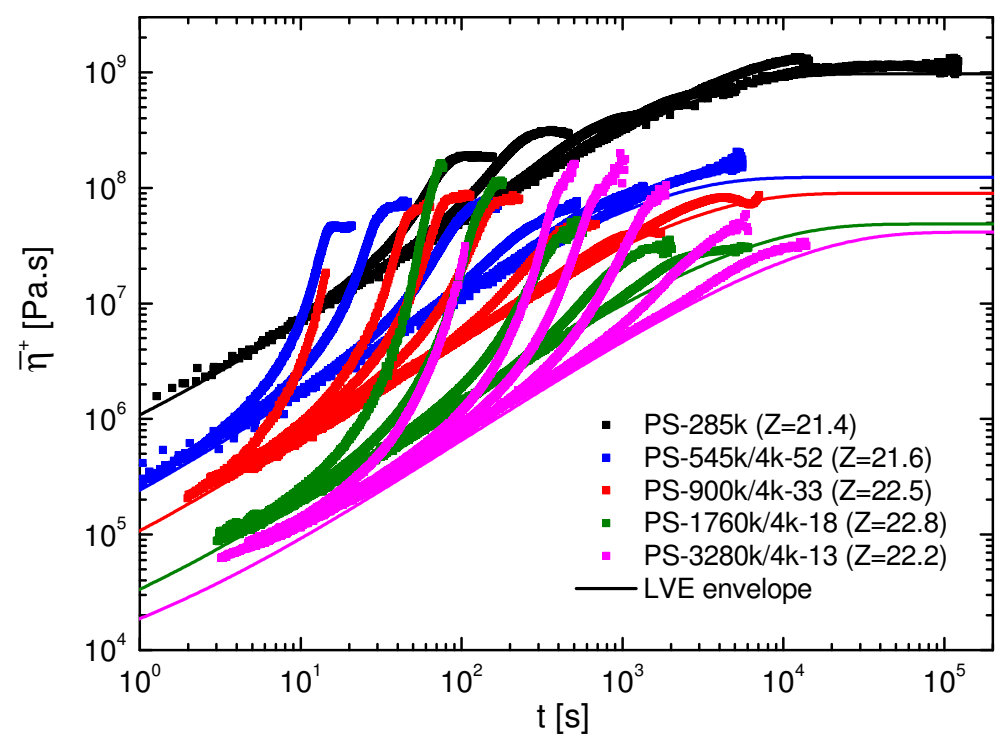

Figure 3: The measured extensional stress growth coefficient as a function of the time for the PS solutions at $130^{\circ} \mathrm{C}$. Data for PS-285k is taken from Ref. [9]. Strain rate (from left to right) for PS-545k/4k-52: $0.2,0.1,0.03,0.01,0.003,0.001 \mathrm{~s}^{-1}$; for PS-900k/4k-33: $0.2,0.07,0.04,0.02,0.007,0.002,0.0007 \mathrm{~s}^{-1}$; for PS-1760k/4k-18: $0.06,0.03,0.01,0.003,0.001 \mathrm{~s}^{-1}$; for PS-3280k/4k-13: $0.03,0.01,0.006,0.003,0.001$, $0.0003 \mathrm{~s}^{-1}$

Figure 3 shows the measured extensional stress growth coefficient $\bar{\eta}^{+}$as a function of time at $130{ }^{\circ} \mathrm{C}$ for all the PS solutions. The solid lines in the figure are predictions from the LVE parameters listed in Table 3. For each solution except PS-3280k/4k-13, $\bar{\eta}^{+}$reaches a clear steady-state value when brittle 


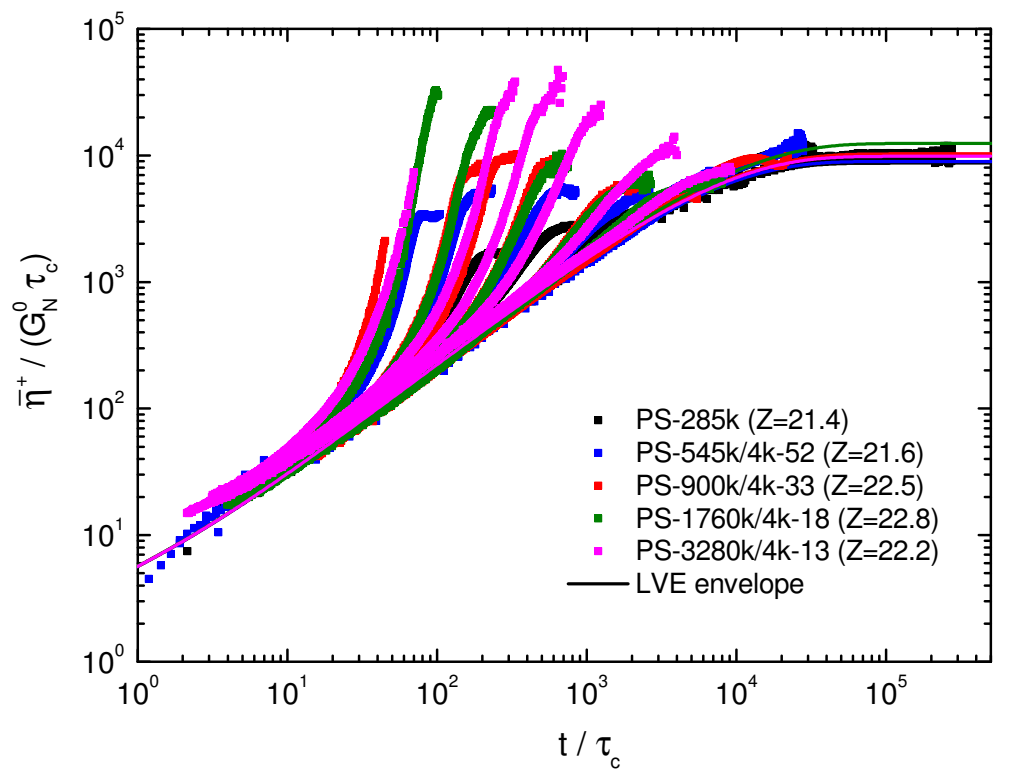

Figure 4: The normalized extensional stress growth coefficient as a function of the normalized time for the PS solutions. Data for PS-285k is taken from Ref. [9]; data for PS-545k/4k-52 is taken from Ref. [10].

fracture does not occur. $\bar{\eta}^{+}$at the high rates for PS-3280k/4k-13 (and also the highest rate for PS$1760 \mathrm{k} / 4 \mathrm{k}-18$ ) tends to a steady-state value at the end of the measurements, but the steady-state viscosity is not very clear even under a large deformation up to Hencky strain 5. In this case we take the highest value of $\bar{\eta}^{+}$as the steady-state viscosity.

Figure 4 compares the solutions under dimensionless parameters which are normalized the same way as we did in Figure 2. The LVE envelopes (solid lines) in the figure overlap each other as expected. For each solution, the normalized transient data also overlaps each other when stretch rate is relatively low. However, at high stretch rate, each solution shows a different behavior. The solution with lower concentration shows obviously higher strain hardening than the solution with higher concentration.

The difference is clearer in Figure 5 where the normalized steady-state viscosity is plotted as a function of the normalized stretch rate. The Rouse time can be estimated as $\tau_{R}=Z^{2} \tau_{c}$ [9]. Since $Z \approx 22$ for all the samples, stretching faster than inverse Rouse time means the normalized stretch rate $\dot{\varepsilon} \tau_{c}>0.002$ in Figure 5. In this region, it is clear that the steady-state viscosity of PS-285k is stretch rate thinning, which agrees with the observations reported by Bach et al. [1] for PS-200k and PS-390k. When the concentration of PS decreases from $100 \%$ to $52 \%$, the trend of the steady-state viscosity changes from stretch rate thinning to stretch rate constant and followed by thinning again. When the concentration further decreases to $33 \%$, a short period of stretch rate thickening is seen, and quickly followed by stretch rate constant and thinning. Finally, when the concentration is lower than $20 \%$, the steady-state viscosity shows stretch rate thickening, which agrees with the observations reported by Bhattacharjee et al. [2] and Sridhar et al. [3] where the highest concentration of PS is $20 \%$.

Since the solutions are compared under normalized parameters in Figure 5, the only parameter that cannot be normalized in the figure seems to be the number of Kuhn segments between entanglements, $N_{e}$, which is proportional to the entanglement molecular weight $M_{e}$. In non-dimensional terms, $N_{e}$ equals the square of the maximum stretch ratio $\lambda_{\max }$ as shown in Table 3 . It seems reasonable that with the increased flexibility associated with a larger value of $N_{e}$ the solution at a lower concentration can reach a higher steady-state viscosity. However, it is not clear if the rather dramatic effect on the 


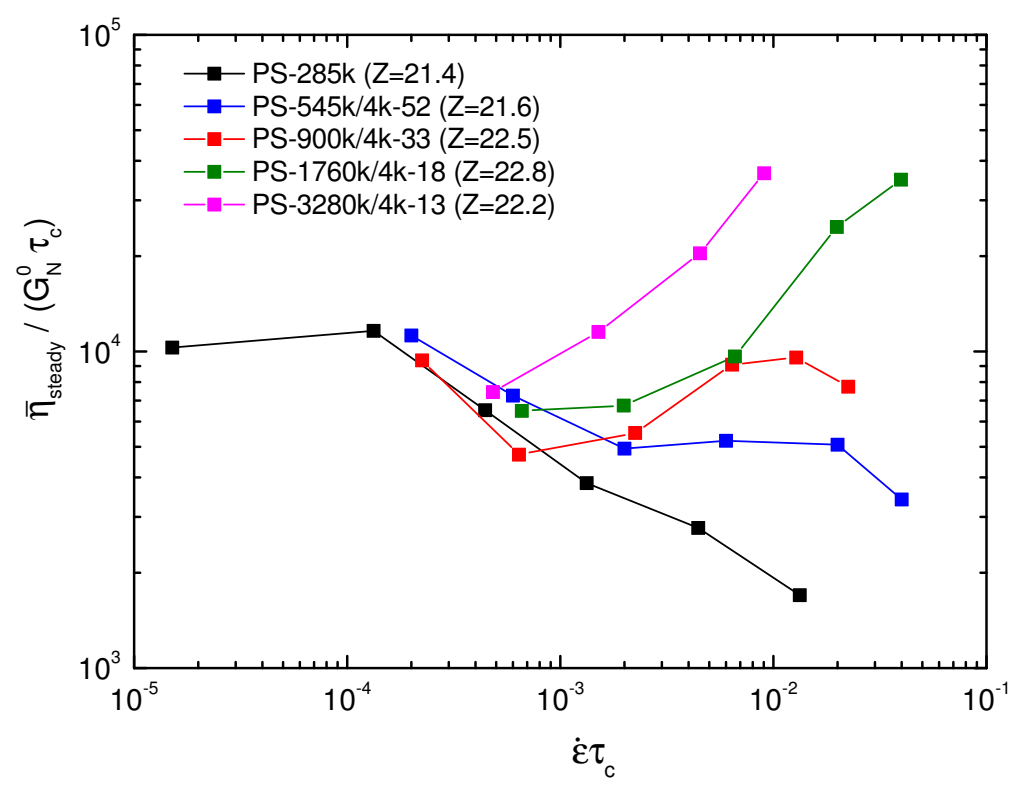

Figure 5: The normalized steady-state viscosity as a function of the normalized stretch rate for the PS solutions. Data for PS-285k is taken from Ref. [9]; data for PS-545k/4k-52 is taken from Ref. [10].

extensional viscosity is associated with this parameter change alone.

Yaoita et al. [11] and Ianniruberto et al. [12] suggest that the monomeric friction is reduced by stretching and orientating the polymer chains during extension. For solutions the average anisotropy is governed by the solvent and remains small, so the friction does not change significantly. But for melts the friction decreases significantly under fast elongation and suppresses the chain stretch, which leads to lower viscosity than expected. According to Yaoita et al., the reduction of friction is less with lower concentration of the polymer, which seems to agree with our observations in this work.

Moreover, in our previous work [10] we have shown that when the same PS is diluted to the same concentration with a range of solvents, the qualitative behavior of the normalized steady-state viscosity is different from one solvent to another. In this case, since $\lambda_{\max }$ is the same, it seems that the only explanation is the reduction of friction due the nematic interactions between both polymer-polymer and polymer-solvent. The solvents used by Bhattacharjee et al. [2] and Sridhar et al. [3] to dilute PS are not oligomeric styrene as we used. But at the PS concentration lower than $20 \%$, the steady-state viscosity has the same trend, which indicates the interaction between polymer-solvent is weaker at low concentration. Furthermore, in the recent work of Masubuchi et al. [17], the authors suggest that the magnitude of orientation/stretch-induced reduction of friction is not universal but changes with the chemical structure of polymers. This may explain why some other polymer melts do not show stretch rate thinning behavior as PS melts, but show stretch rate thickening as PS solutions.

\section{Conclusions}

We have shown that the PS solutions which have identical linear rheology behave differently in extensional nonlinear rheology. In fact, we observed a systematic change of the extensional steady-state viscosity from stretch rate thinning to stretch rate thickening by decreasing the PS concentration. It is suggested that the parameter which dominates the nonlinear behavior is the orientation/stretch-induced reduction of friction reported by Yaoita et al. [11] and Ianniruberto et al. [12]. But more work will be 
needed for a full understanding.

\section{Appendix}

The LVE properties of the PS solutions and the styrene oligomer OS-4k were obtained from small amplitude oscillatory shear (SAOS) measurements. An $8 \mathrm{~mm}$ plate-plate geometry was used on an ARES-G2 rheometer from TA instruments. The measurements were performed at a range of temperatures under nitrogen. For each sample, the data was shifted to a single master curve at the reference temperature $T_{r}=130{ }^{\circ} \mathrm{C}$ using the time-temperature superposition procedure. The temperature shift factor $a_{T}$ is reported in Table 4 for each PS solution. Steady shear flow measurement was also performed for OS-4k at $130{ }^{\circ} \mathrm{C}$ using the $8 \mathrm{~mm}$ plate-plate geometry on the ARES-G2 rheometer. The zero-shear-rate viscosity for OS- $4 \mathrm{k}$ is $\eta_{s}=248 \mathrm{~Pa}$.s.

Figure 6 shows the result of SAOS measurement for the solvent OS-4k. At a wide frequency range up to $\omega \approx 1 \times 10^{4} \mathrm{rad} / \mathrm{s}$, OS-4k behaves like a Newtonian fluid and the loss modulus $G^{\prime \prime}$ matches $\eta_{s} \omega$ where $\eta_{s}$ is obtained from steady shear flow measurement. The relaxation time of OS-4k is in the order of $10^{-5} \mathrm{~s}$ at $130^{\circ} \mathrm{C}$.

Table 4: The shift factor $a_{T}$ from different temperatures to $T_{r}=130{ }^{\circ} \mathrm{C}$ for the polystyrene solutions. $a_{T g}$ is the shift factor calculated by Eq. 3. For the melts $a_{T g}=1$

\begin{tabular}{lccccc}
\hline \hline Sample Name & $120^{\circ} \mathrm{C}$ & $150{ }^{\circ} \mathrm{C}$ & $170{ }^{\circ} \mathrm{C}$ & $190{ }^{\circ} \mathrm{C}$ & $a_{T g}$ \\
\hline PS-545k/4k-52 & - & 0.035 & 0.0031 & - & 0.145 \\
PS-900k/4k-33 & - & 0.042 & 0.0042 & - & 0.0408 \\
PS-1760k/4k-18 & 7.59 & 0.050 & 0.0052 & 0.0011 & 0.0237 \\
PS-3280k/4k-13 & - & 0.054 & 0.0071 & 0.0013 & 0.0214 \\
\hline \hline
\end{tabular}

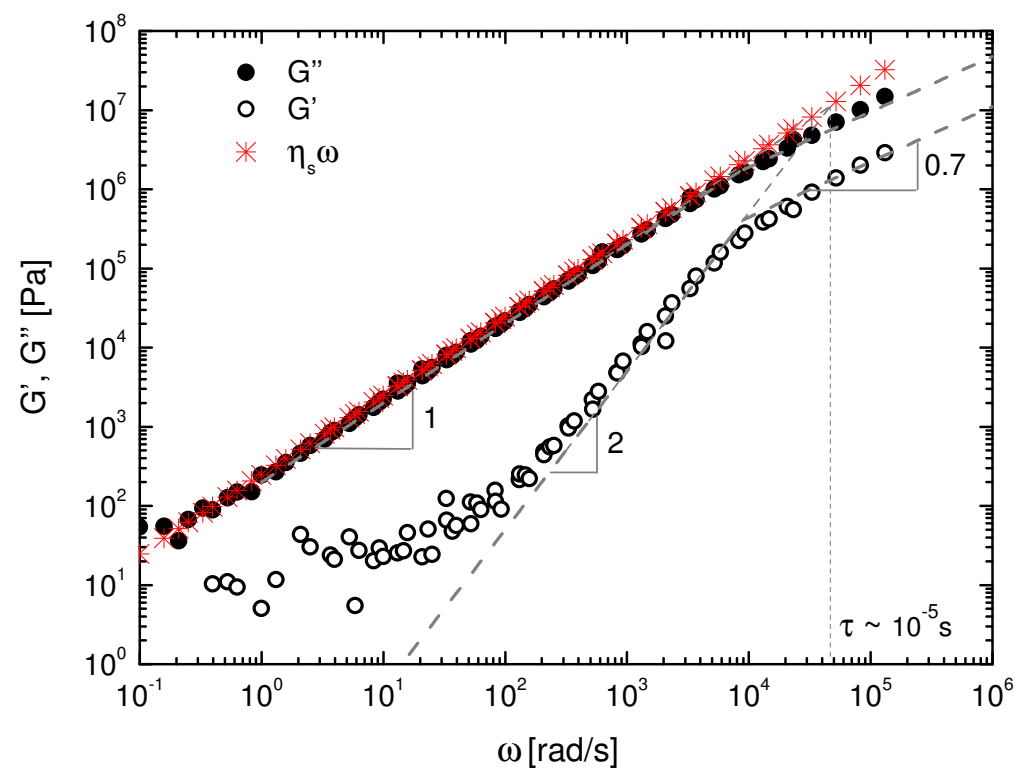

Figure 6: Storage modulus and loss modulus of OS-4k as a function of angular frequency at $130^{\circ} \mathrm{C}$.

The temperature shift factor $a_{T}$ for PS melts can be described by the Williams-Landel-Ferry (WLF) equation 


$$
\log _{10} a_{T}=\frac{-c_{1}^{0}\left(T-T_{r}\right)}{c_{2}^{0}+\left(T-T_{r}\right)},
$$

where $c_{1}^{0}=8.99, c_{2}^{0}=81.53 \mathrm{~K}, T_{r}=130^{\circ} \mathrm{C}$, and $T$ is temperature in ${ }^{\circ} \mathrm{C}$ [18]. However, with the same reference temperature $T_{r}=130^{\circ} \mathrm{C}$, Eq. 2 can not describe the PS solutions. This is because the melts and solutions have different values of $T_{g}$ and therefore $T_{r}-T_{g}$ is different. The effect of different $T_{g}$ can be corrected by a shift factor $a_{T g}$ which can also be obtained from a WLF equation as reported by Ref.[15]:

$$
\log _{10} a_{T g}=\frac{-c_{1}^{0} \Delta T_{g}}{c_{2}^{0}+\Delta T_{g}},
$$

where $c_{1}^{0}$ and $c_{2}^{0}$ have the same values as in Eq.2 and $\Delta T_{g}$ is the difference of $T_{g}$ between PS melts and solutions. Figure 7 plots the corrected shift factor $a_{T} a_{T g}$ as a function of $T-T_{g}$. A mater curve is obtained for all the PS solutions and melts, which agrees with the observations reported in Ref. [15]. Values of $a_{T g}$ for the solutions are listed in Table 4.

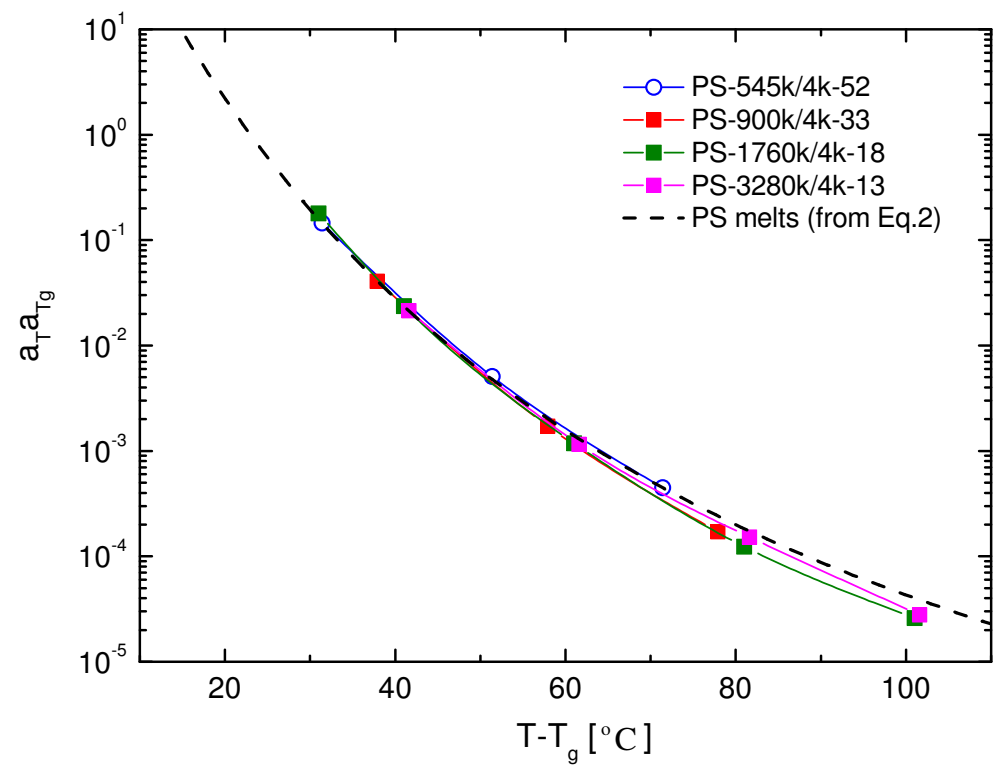

Figure 7: Corrected temperature shift factor $a_{T} a_{T g}$ as a function of $T-T_{g}$. For PS melts $a_{T g}=1$ at the reference temperature $T_{r}=130^{\circ} \mathrm{C}$.

The relationship between the solution plateau modulus, $G_{N}^{0}(\phi)$, and the melt plateau modulus, $G_{N}^{0}(1)$, is given by

$$
G_{N}^{0}(\phi)=G_{N}^{0}(1) \phi^{1+\alpha},
$$

where $\alpha$ is the dilution exponent. It has been reported by Huang et al. [9] that $\alpha=1$ for highly concentrated PS solutions with $\phi>0.4$. Figure 8 plots $G_{N}^{0}$ as a function of $\phi$ for all the samples listed in Table 3. It seems that $\alpha=1$ also works for the PS solutions with lower concentrations at least down to $\phi=0.18$. There is a deviation from $\alpha=1$ for PS-3280k/4k-13 which has the lowest concentration of $\phi=0.13$. However, considering the higher polydispersity of the sample and the less satisfactory BSW fitting as shown in Figure 1, this deviation might be an artifact. In addition to the samples listed in Table 3, we have also diluted PS-545k in OS-4k with different concentrations down to $\phi=0.17$. LVE 


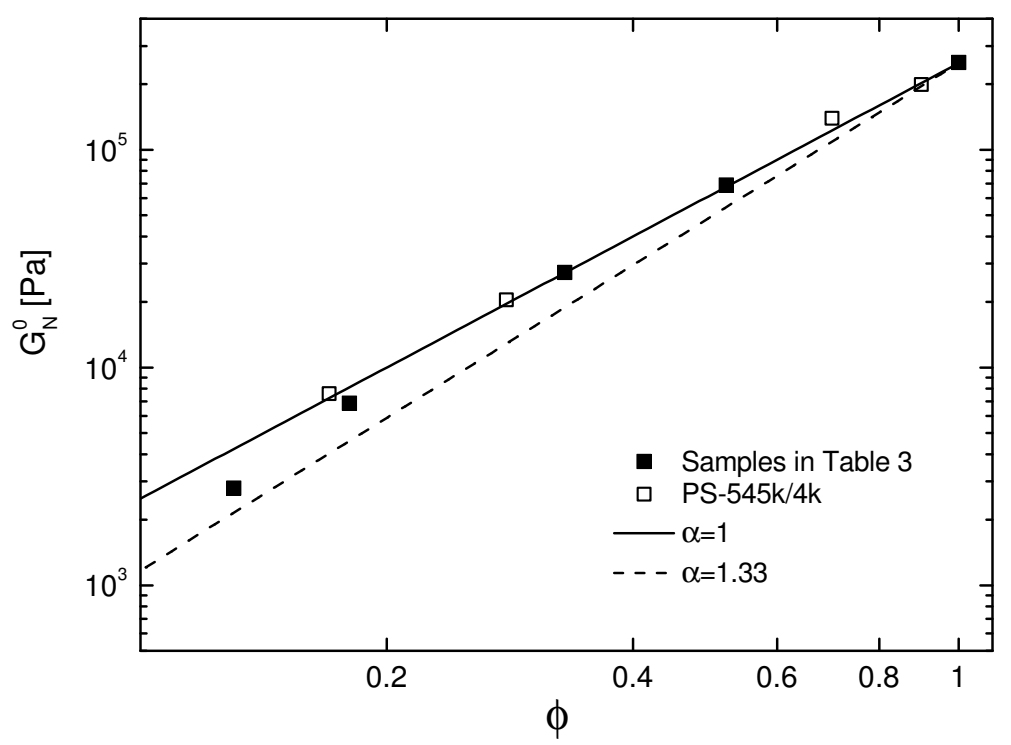

Figure 8: Plateau modulus $G_{N}^{0}$ as a function of the polymer volume fraction $\phi$. The slope of the solid line in the figure is $2(\alpha=1)$, and the slope of the dashed line is $2.33(\alpha=4 / 3)$.

measurements and BSW fittings were also performed for these samples. The plateau modulus $G_{N}^{0}$ as a function of $\phi$ for the additional samples PS-545k/4k is also shown in Figure 8 as open symbols. All of them follow $\alpha=1$.

\section{Acknowledgments}

The research leading to these results has received funding from the Danish National Advanced Technology Foundation under LANI on Grant 011-2011-3, and the Danish Council for Independent Research - Natural Sciences under EPMEF on Grant 0602-02179B. We thank Andriy Dorokhin from DTU Nanotech for performing SEC. 


\section{References}

[1] Bach, A.; Almdal, K.; Rasmussen, H. K.; Hassager, O. Macromolecules 2003, 36, 5174-5179.

[2] Bhattacharjee, P. K.; Oberhauser, J. P.; McKinley, G. H.; Leal, L. G.; Sridhar, T. Macromolecules 2002, 35, 10131-10148.

[3] Sridhar, T.; Acharya, M.; Nguyen, D. A.; Bhattacharjee, P. K. Macromolecules 2014, 47, 379-386.

[4] Doi, M.; Edwards, S. F. The Theory of Polymer Dynamics; Oxford Univ. Press: New York, 1986.

[5] Daoud, M.; de Gennes, P.G. J. Polym. Sci. Polym. Phys. 1979, 17, 1971-1981

[6] Doi, M. J. Polym. Sci.: Polym. Lett. 1981, Ed. 19, 265-273.

[7] Marrucci, G.; Grizzuti, N. Gazz. Chim. Ital. 1988, 118, 179-185.

[8] Marrucci, G.; Ianniruberto, G. J. Non-Newtonian Fluid Mech. 1996, 65, 241-246.

[9] Huang, Q.; Mednova, O; Rasmussen, H. K.; Alvarez, N. J.; Skov, A. L.; Almdal, K.; Hassager, O. Macromolecules 2013, 46, 5026-5035.

[10] Huang, Q.; Alvarez, N. J.; Matsumiya,Y.; Rasmussen, H. K.; Watanabe, H.; Hassager, O. ACS Macro Lett. 2013, 2, 741-744.

[11] Yaoita, T.; Isaki, T.; Masubuchi, Y.; Watanabe, H.; Ianniruberto, G.; Marrucci, G. Macromolecules $2012,45,2773-2782$.

[12] Ianniruberto, G.; Brasiello, A.; Marrucci, G. Macromolecules 2012, 45, 8058-8066.

[13] Baumgaertel, M.; Schausberger, A.; Winter, H. H. Rheol. Acta 1990, 29, 400-408.

[14] Jackson, J. K.; Winter, H. H. Macromolecules 1995, 28, 3146-3155.

[15] Wagner, M. H. Rheol Acta 2014, 53, 765-777

[16] Bach, A.; Rasmussen,H. K.; Hassager, O. J. Rheol. 2003, 47, 429-441.

[17] Masubuchi, Y.; Matsumiya, Y.; Watanabe, H. Macromolecules 2014, 47, 6768-6775.

[18] Huang, Q. Ph.D. thesis, Technical University of Denmark, 2013 